\section{Das Erfordernis der dreijährigen Tätigkeit im Rahmen des Verzichts zu Gunsten der Anstellung bei Vertragsarzt oder MVZ}

\section{Einleitung}

Die Bundesrepublik Deutschland steht in den nächsten Jahren vor einem erheblichen demographischen Wandel. In den nächsten Jahren wird die Babyboomer-Generation in den Ruhestand bzw. Rente gehen. ${ }^{1}$ Die gleiche Altersentwicklung zeigt sich in der vertragsärztlichen Versorgung. Das Durchschnittsalter der Ärzte und Psychotherapeuten in der vertragsärztlichen Versorgung ist in den vergangenen zehn Jahren von rund 52,7 (2011) auf 54,2 (2020) Jahre gestiegen. $^{2}$

Mit dem nahenden Ruhestand geht für Vertragsärzte die Überlegung der Praxisübergabe bzw. der wirtschaftlichen Verwertung der Praxis einher. Zum wesentlichen Unternehmenswert einer Praxis gehört im Rahmen des immateriellen Werts unter anderem insbesondere die Zulassung zur vertragsärztlichen Versorgung gemäß §95 Abs. 2 S. 1 SGB V. Dies ist dann von wesentlicher Bedeutung, wenn für die Arztgruppe des abgebenden Vertragsarztes gemäß §103 Abs. 1 S. 1 SGB V Zulassungsbeschränkungen angeordnet sind. Denn dann kann der übernehmende Arzt oder Psychotherapeut die Zulassung zur vertragsärztlichen Versorgung nicht im einfachen Antragsverfahren erlangen.

Zur „Übertragung“ der Zulassung auf eine andere Rechtsperson bestehen in diesem Fall zwei rechtliche Möglichkeiten. Entweder der Vertragsarzt stellt gemäß $§ 103$ Abs. 3a S. 1 SGB V einen Antrag auf Durchführung des Nachbesetzungsverfahrens gemäß § 103 Abs. 4 SGB V und verzichtet anschließend auf seine Zulassung oder der Vertragsarzt verzichtet auf seine Zulassung, um gemäß § 103 Abs. 4a, 4b S. 1 SGB V bei einem anderen Vertragsarzt oder einem

1 Vgl. nur Ausblick auf die Bevölkerungsentwicklung in Deutschland und den Bundesländern nach dem Corona-Jahr 2020, Statistisches Bundesamt 2021, S. $19 \mathrm{ff}$.

2 Statistische Informationen aus dem Bundesarztregister, Bundesgebiet insgesamt, Stand: 31.12.2020, S. 16
Medizinischen Versorgungszentrum (MVZ) als angestellter Arzt tätig zu werden.

Beim Nachbesetzungsverfahren entscheidet der Zulassungsausschuss zunächst gemäß § 103 Abs. 3a SGB V, ob ein Nachbesetzungsverfahren überhaupt durchgeführt wird (1. Stufe des Nachbesetzungsverfahrens). Ergeht hierzu ein positiver Beschluss, schreibt die Kassenärztliche Vereinigung gemäß § 103 Abs. 4 S. 1 SGB V den Vertragsarztsitz aus und stellt eine Liste der eingehenden Bewerbungen zusammen (2. Stufe des Nachbesetzungsverfahrens). In einem weiteren Schritt hat der Zulassungsausschuss gemäß $\S 103$ Abs. 4 S. 4 SGB $V$ unter mehreren Bewerbern den Nachfolger für den Vertragsarztsitz des abgebenden Arztes nach pflichtgemäßem Ermessen auszuwählen (3. Stufe des Nachbesetzungsverfahrens).

Demgegenüber ist beim Verzicht zu Gunsten der Anstellung gerade kein Nachbesetzungsverfahren notwendig. Der Zulassungsausschuss hat die Anstellung des abgebenden Arztes gemäß §103 Abs. 4a, 4b S. 1 SGB V zu genehmigen, wenn Gründe der vertragsärztlichen Versorgung dem nicht entgegenstehen. Auf die Genehmigung der Anstellung besteht ein Rechtsanspruch. ${ }^{3}$ Dies bedeutet, dass der Vertragsarztsitz des abgebenden Arztes weder durch die Kassenärztliche Vereinigung auszuschreiben ist, noch hat der Zulassungsausschuss eine Auswahlentscheidung hinsichtlich der Person, auf den sich die Anstellungsgenehmigung gemäß §95 Abs. 2 S. 7, 9 S. 1 SGB V bezieht. Eine spätere Nachbesetzung der Arztstelle ist - ohne eine Auswahlentscheidung des Zulassungsausschusses - möglich, auch wenn Zulassungsbeschränkungen angeordnet sind, $\S 95$ Abs. 4a S. 5, 4b S. 5 SGB V.

3 Ladurner, Ärzte-ZV, Zahnärzte-ZV, 2017, $\S 103$ SGB V Rn. 94.

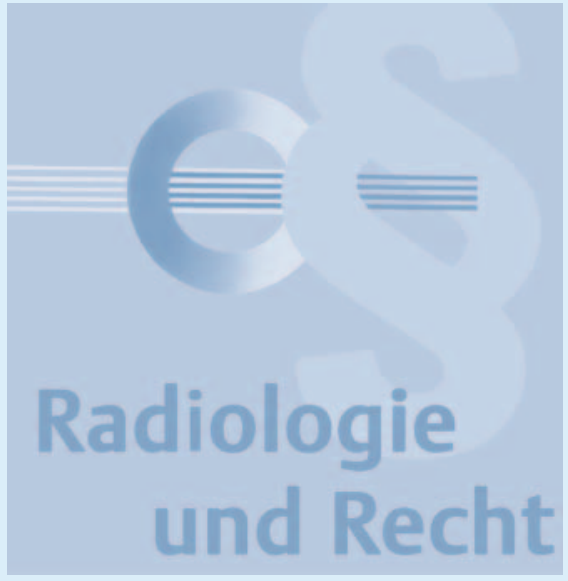

Vor dem Urteil des Bundesozialgerichts (BSG) vom 04.05.2016, Az.: B 6 KA 21/15R setzten die Zulassungsausschüsse regelmäßig eine bestimmte Dauer der Tätigkeit des Verzichtenden - zumeist ca. 2 Quartale als sogenannte „Schamfrist“ - voraus, bevor die Arztstelle nachbesetzt werden konnte. ${ }^{4}$ Teilweise wurde jedoch auch eine Nachbesetzbarkeit nach „einer logischen Sekunde“ als zulässig erachtet. ${ }^{5}$

Das BSG hatte in dem Urteil vom 04.05.2016 6 entschieden, dass das Recht zur Nachbesetzung einer Arztstelle grundsätzlich nur dann bestehe, wenn der Arzt dort mindestens drei Jahre tätig war, oder - wenn er früher ausscheidet - jedenfalls ursprünglich die Absicht hatte, dort mindestens drei Jahre tätig zu sein (die „DreiJahres-Frist“). ${ }^{7}$ Die Entscheidung betraf nach dem Sachverhalt nur ein MVZ. Die hierbei aufgestellten Rechtsgrundsätze sind jedoch auf Berufsausübungsgemeinschaften (BAG) zu übertragen. ${ }^{8}$

Sinn und Zweck der gleich darzustellenden Anforderungen des BSG war es, Gestaltungsmissbrauch im Sinne einer Umgehung

4 Gerdts, Das Urteil des BSG vom 04.05.2016 B 6 KA 21/15 R - Auswirkungen auf die Vertragsgestaltung bei Praxisübertragungen, ZMGR 2018, 9, 10.

5 Greve, Anmerkung zum Urteil des BSG vom 04.05.2016, Az.: B 6 KA 21/15 R, ZMGR 2016, 379.

6 BSG, Urteil vom 04.05.2016, Az.: B 6 KA 21/ 15 R.

7 BSG, Urteil vom 04.05.2016, Az.: B 6 KA 21/ $15 \mathrm{R}$.

8 Greve, ZMGR 2016, 379; Willaschek, Anmerkung zu BSG, Urtl. V. 4.5.2016 - B 6 KA 21/ 15 R (Bay. LSG), MedR 2016, 1011. 
des Nachbesetzungsverfahrens zu verhindern. ${ }^{9}$ Dies ist bei der Bewertung des Urteils und den nachfolgenden Ausführungen im Hinterkopf zu behalten. Die Drei-JahresFrist beruht ausschließlich auf Richterrecht und ist im Gesetz jedenfalls nicht ausdrücklich normiert.

Dies vorausgeschickt soll nachfolgend die rechtliche Begründung der Drei-JahresFrist, deren grundsätzliche Voraussetzungen und Ausnahmen von derselben dargelegt und hierzu kritisch Stellung genommen werden.

\section{Herleitung und Gründe der Drei-Jahres-Frist}

\section{$\S 103$ Abs. 4a S. 1 SGB V normiert:}

„Verzichtet ein Vertragsarzt in einem Planungsbereich, für den Zulassungsbeschränkungen angeordnet sind, auf seine Zulassung, um in einem medizinischen Versorgungszentrum tätig zu werden, so hat der Zulassungsausschuss die Anstellung zu genehmigen, wenn Gründe der vertragsärztlichen Versorgung dem nicht entgegenstehen; eine Fortführung der Praxis nach Absatz 4 ist nicht möglich."

Systematisch ist zwischen dem Verzicht des Vertragsarztes auf seine Zulassung und der dem MVZ zu erteilenden Genehmigung zur Anstellung gerade dieses Arztes zu unterscheiden. ${ }^{10}$ Das BSG begründete die DreiJahres-Frist insbesondere mit dem Ziel des verzichtenden Arztes, in dem $M V Z^{11}$ tätig zu werden, aus dem Ausnahmecharakter der Regelung des $§ 103$ Abs. 4a S. 1 SGB V und systematischen Erwägungen, welche

9 Schroeder/Printzen in: Ratzel/Luxemburger, Handbuch Medizinrecht, 4. Aufl., Heidelberg 2021, Rn. 754; Willaschek, MedR 2016, 1011,1012 .

10 BSG, Urteil vom 04.05.2016, Az.: B 6 KA 21/ 15 R, Rn. 18; BT-Drucks 15/1525 S. 112; Wenner, Angestellte Ärzte in Medizinischen Versorgungszentren (MVZ) - Verständigungsprobleme zwischen Arbeitsrecht und Vertragsarztrecht? In: FS Preis, München 2021, 1485, 1491 (Fn. 29).

11 Die Entscheidung des BSG hatte einen Verzicht zugunsten eines MVZ nach § 103 Abs. 4a S. 1 SGB V zum Gegenstand. Auf den Verzicht zugunsten eines Vertragsarztes nach $\S 103$ Abs. 4b S. 1 SGB V sind die Ausführungen übertragbar. sogleich zu skizzieren sind. ${ }^{12}$ Das BSG ging zunächst auf den Unterschied zwischen dem Nachbesetzungsverfahren und dem Verzicht zu Gunsten der Anstellung ein.

Für den Fall, dass der Vertragsarzt auf seine Zulassung zu Gunsten der Anstellung verzichte, sei weder eine Entscheidung des Zulassungsausschusses zum „ob“ der Nachbesetzung noch eine Auswahlentscheidung zwischen mehreren Bewerbern um die Praxisnachfolge vorgesehen. Vielmehr sei dem Leistungserbringer die Genehmigung zur Anstellung des Arztes zu erteilen, der auf seine Zulassung verzichtet hat. Entscheidende Voraussetzung für diese Privilegierung sei nach §103 Abs. 4a S. 1 SGB V, dass der Arzt auf seine Zulassung verzichtet habe, „um in einem medizinischen Versorgungszentrum tätig zu werden“. Der entscheidende Unterschied zum Nachbesetzungsverfahren und der Grund dafür, dass die Anstellungsgenehmigung beim Vorliegen der gesetzlichen Voraussetzungen zu erteilen sei, ohne dass dem Zulassungsausschuss ein Entscheidungsspielraum verbleibt, liege darin, dass der Vertragsarzt bei dieser Variante des Zulassungsverzichts seine Tätigkeit innerhalb des Systems der vertragsärztlichen Versorgung und nur mit einem anderen Status dem des Angestellten - fortführt. Diese Gestaltung des Verzichts auf die Zulassung sei deshalb von vornherein auf eine Weiterführung der Versorgung und nicht auf ein Ausscheiden aus dem System angelegt. ${ }^{13}$

Dies ist das Kernargument des BSG zur Rechtfertigung der Dreijahresfrist. Sinn und Zweck des Verzichts zu Gunsten der Anstellung sei einzig ein formaler Statuswandel (Selbstständigkeit in Anstellung). ${ }^{14}$ Der Vorsitzende des 6. Senates merkte im Nachgang auf die Entscheidung an, dass deshalb alle Überlegungen, eine längere Mitarbeit im MVZ sei „häufig nicht im Interesse des Vertragsarztes, der typischerweise seine Tätigkeit zurückfahren oder

12 BSG, Urteil vom 04.05.2016, Az.: B 6 KA 21/ 15 R, Rn 27

13 BSG, Urteil vom 04.05.2016, Az.: B 6 KA 21/ 15 R, Rn 24.

14 Ladurner/Walter/Jochimsen, Rechtsgutachten Stand und Weiterentwicklung der gesetzlichen Regelungen zu medizinischen Versorgungszentren (MVZ), S. 67. beenden möchte“15, von vornherein dem Einwand ausgesetzt seien, dass bei dieser Intention der „falsche“ Weg des Ausscheidens aus der vertragsärztlichen Versorgung gewählt worden sei. ${ }^{16}$

Nach Auffassung des BSG können MVZ und Vertragsärzte die Privilegien, die $§ 103$ Abs. 4a und 4b SGB V im Rahmen der Nachbesetzung vermittelten, grundsätzlich nur in Anspruch nehmen, wenn und soweit der Arzt auf seine Zulassung gerade mit dem Ziel verzichte, selbst in dem MVZ als angestellter Arzt tätig zu werden; es werde also Personenidentität zwischen dem auf die Zulassung verzichtenden Arzt und dem Arzt vorausgesetzt, der die Anstellung in dem MVZ aufnimmt. Die Anstellung eines anderen Arztes als desjenigen, der auf die Zulassung verzichtet habe, sei nicht Gegenstand des $\S 103$ Abs. 4a Satz 1 SGB V, sondern des $\S 103$ Abs. 4c SGB V, der eine Auswahlentscheidung der Zulassungsgremien vorsehe. ${ }^{17}$

Der Gesetzgeber habe durch das GKV-VStG in $\S 103$ Abs. 3a Satz 7 SGB V eine Regelung zum Abbau der Überversorgung geschaffen. Der Anwendungsbereich dieser Regelung dürfe nach Auffassung des BSG nicht durch eine erweiternde, über den Wortlaut hinausgehende Auslegung der Ausnahmeregelung des $\S 103$ Abs. 4a SGB V eingeschränkt werden. Derartige Ausnahmeregelungen seien daher eng auszulegen. ${ }^{18}$

Zusammenfassend kann daher nach dem BSG nicht jede beliebig geringe Dauer einer angestrebten Angestelltentätigkeit den Anspruch auf die Erteilung einer Genehmigung nach dieser Vorschrift begründen. Dies folgt daraus, dass der Arzt auf seine Zulassung verzichtet, mit dem Ziel gerade bei dem Vertragsarzt oder dem MVZ

15 Preis, Das „Dreijahresdogma“ im Spannungsfeld von Sozialrecht und Arbeitsrecht, GesR 2018, 75, 79 .

16 Wenner, Mehr Chancen oder mehr Risiken für die vertragsärztliche Versorgung? Eine Zwischenbilanz zu den Medizinischen Versorgungszentren (MVZ) am Ende der 19. Legislaturperiode des Bundestages, SGb 2021, 593 , 601.

17 BSG, Urteil vom 04.05.2016, Az.: B 6 KA 21/ $15 \mathrm{R}, \mathrm{Rn} 25$.

18 BSG, Urteil vom 04.05.2016, Az.: B 6 KA 21/ $15 \mathrm{R}, \mathrm{Rn} 26$ 
angestellt zu werden. Daneben sprächen systematische Erwägungen dafür und die Vorschriften des $\S 103$ Abs. 4a S. 1 und 4b S. 1 SGB $V$ sind als Ausnahmevorschriften eng auszulegen.

\section{Umfang und schrittweise Verringerung der dreijährigen Tätigkeit}

Nachdem zunächst die dogmatischen Erwägungen für eine gewisse Mindestdauer der Tätigkeit eines verzichtenden Arztes bei dem anstellenden Leistungserbringer aufgezeigt wurden, ist zu untersuchen, mit welcher Dauer und in welchem zeitlichen Umfang (Arbeitsstunden) der Arzt bei dem anstellenden Leistungserbringer tätig sein muss. Darüber hinaus soll beleuchtet werden, unter welchen Voraussetzungen der verzichtende Arzt den zeitlichen Umfang seiner Tätigkeit reduzieren kann.

Das BSG orientierte sich zur Bemessung der dreijährigen Tätigkeit an $\S 103$ Abs. 3a S. 5, S. 3, Abs. 4 S. 5 Nr. 6 SGB V und folgte der teilweise von der Literatur vertretenen Ansicht, dass eine Tätigkeit von 1 bis 2 Quartalen (Dauer der Tätigkeit) ausreichend sei, hierbei nicht. Als innerer Vorgang sei die Absicht des auf die Zulassung verzichtenden Arztes, bei dem Leistungserbringer tätig zu werden, einer objektiven Überprüfung nicht ohne Weiteres zugänglich. Nach Ablauf von drei Jahren der Tätigkeit eines angestellten Arztes im MVZ könne aber davon ausgegangen werden, dass die gesetzlich vorgegebene Gestaltung auch tatsächlich gewollt und gelebt worden sei. ${ }^{19}$

Aus dem Zusammenhang zwischen dem Verzicht auf die Zulassung und der Erteilung der Anstellungsgenehmigung folge, dass nach dem Verzicht auf eine hälftige Zulassung i. S. d. § 19a Abs. 2 Ärzte-ZV keine Anstellungsgenehmigung für eine ganze Stelle erteilt werden könne. Umgekehrt könne der Umfang der Tätigkeit, für die eine Anstellungsgenehmigung erteilt werde, aber hinter dem Umfang der Zulassung zurückbleiben, weil damit kein Anstieg, sondern

19 BSG, Urteil vom 04.05.2016, Az.: B 6 KA 21/ 15 R, Rn. 28. ein - bedarfsplanungsrechtlich erwünschter - Abbau der Überversorgung bewirkt werde. ${ }^{20}$

Das BSG knüpft also für den Versorgungsauftrag der zu erteilenden Anstellungsgenehmigung nach dem Verzicht zu Gunsten der Anstellung an den Versorgungsauftrag der Zulassung an (zeitlicher Umfang der Tätigkeit). Der Versorgungsauftrag der Anstellungsgenehmigung darf insoweit bedarfsplanungsrechtlich nicht über den Versorgungsauftrag der Zulassung des Arztes hinausausgehen, kann jedoch grundsätzlich dahinter zurückbleiben. Bleibt der Anrechnungsfaktor der Anstellungsgenehmigung hinter dem Versorgungsauftrag der Zulassung zurück, auf die der Arzt zu Gunsten der Anstellung verzichtet hatte, kann die Stelle nach dem späteren Ausscheiden des Arztes jedoch auch nur im Umfang des Anrechnungsfaktors der erteilten Anstellungsgenehmigung nachbesetzt werden. ${ }^{21}$

Das BSG gesteht solchen Ärzten, die zwar nach den dargestellten Grundsätzen tatsächlich bei einem Leistungserbringer tätig werden wollen, aber altersbedingt ihren Tätigkeitsumfang allmählich vermindern wollen, eine entsprechende Ausnahme zu (schrittweise Verringerung der Tätigkeit; auch Abschmelzen genannt). Für diese Ärzte kann sich die angestrebte Anstellung für eine Dauer von wenigstens drei Jahren als zentraler Indikator für die Tätigkeitsabsicht im Sinne des § 103 Abs. 4a Satz 1 SGB $\checkmark$ nur auf die Tätigkeit als solche beziehen, aber nicht gänzlich auch auf den zeitlichen Umfang. Ein solcher Arzt muss daher zunächst ein Jahr in dem Umfang bei dem Leistungserbringer tätig sein, in denen er zuvor als zugelassener Arzt an der Versorgung teilgenommen hat. Sodann kann er seinen Beschäftigungsumfang in den beiden folgenden Jahren aber vermindern, etwa indem er jeweils seinen Beschäftigungsumfang schrittweise um den Anrechnungsfaktor 0,25 reduziert. Dies wirkt sich in der Folge auch nicht negativ auf das

20 BSG, Urteil vom 04.05.2016, Az.: B 6 KA 21/ 15 R, Rn. 18.

21 BSG, Urteil vom 04.05.2016, Az.: B 6 KA 21/ 15 R, Rn. 11.
Nachbesetzungsrecht des anstellenden Leistungserbringers aus. ${ }^{22}$

Obwohl die Formulierung der Rechtsprechung die Reduzierung des Tätigkeitsumfangs von einer Anstellungsgenehmigung mit vollem Versorgungsauftrag im Wortlaut nach als Beispiel aufgezeigt werden sollte, halten einige Kassenärztliche Vereinigung die genannte Reduktionsmöglichkeit für die einzig zulässige. ${ }^{23}$

Ob eine Reduktion des Tätigkeitsumfangs eines Arztes dessen Anstellung mit halbem Versorgungsauftrag genehmigt wurde, bereits nach einem Jahr um den Anrechnungsfaktor von 0,25 zulässig sein soll, wird daher nicht einheitlich beurteilt. Da das BSG nach dem Wortlaut seiner Entscheidung ausdrücklich die Interessen der Ärzte, die altersbedingt ihren Tätigkeitsumfang allmählich vermindern wollen, berücksichtigen wollte, spricht nichts gegen die Zulässigkeit einer Reduktion. Ganz im Gegenteil würde eine gegenteilige Auslegung der Entscheidung des BSG gegen den Wortlaut und Telos derselben verstoßen.

Offen ist jedoch gleichwohl, ab welchem Alter Vertragsärzte, die zu Gunsten der Anstellung verzichten, von dieser Reduktionsmöglichkeit Gebrauch machen können.

\section{Rechtliche Folge des Verstoßes gegen die Drei-Jahres-Frist}

Endet die Tätigkeit des Arztes, der zu Gunsten der Anstellung auf seine Zulassung verzichtet hatte, vor Ablauf von drei Jahren, hängt das Nachbesetzungsrecht des Leistungserbringers nach dem BSC davon ab, ob nach den Umständen davon ausgegangen werden kann, dass der ursprünglich zugelassene Arzt zunächst tatsächlich zumindest drei Jahre beim Leistungserbringer tätig werden wollte, diese Absicht aber aufgrund von Umständen, die ihm zum Zeitpunkt des Verzichts auf die Zulassung

22 BSG, Urteil vom 04.05.2016, Az.: B 6 KA 21/ 15 R, Rn. 30.

23 Pflugmacher, Aktuelle Rechtsfragen zum GKVVSG und dem „3-Jahres-Urteil“ des BSG aus Sicht der Krankenhäuser, KrV 2017, 107, 113. 
noch nicht bekannt waren, nicht mehr realisieren konnte. ${ }^{24}$

Die rechtliche Folge der vom BSG aufgestellten Grundsätze zeigt sich also nicht bei der Genehmigung der Anstellung selbst. Erst wenn die Arztstelle das erste Mal nachbesetzt werden soll und die Dreijahresfrist nicht eingehalten wurde, kann sich ein Ausschluss der Nachbesetzbarkeit der Arztstelle für den anstellenden Vertragsarzt oder das MVZ ergeben. Dies gilt jedoch dann nicht, wenn eine zulässige Ausnahme von den vom BSG aufgestellten Grundsätzen einschlägig ist (vgl. V.). Erst zu diesem Zeitpunkt muss dann anhand der vorliegenden Erkenntnisse eine Beurteilung erfolgen, ob die Absicht für eine zumindest dreijährige Tätigkeit ursprünglich vorgelegen hat. ${ }^{25}$

Sofern eine Arztstelle, die aus dem Verzicht zu Gunsten der Anstellung gemäß $§ 103$ Abs. 4a S. 1, 4b S. 1 SGB V entstanden ist, bereits einmal bestandskräftig nachbesetzt wurde, ist die Rechtsposition des betroffenen anstellenden Leistungserbringers jedoch schutzwürdig. Nach dem BSG kann auf eine bestandskräftig erteilte Anstellungsgenehmigung im Regelfall auch eine darauffolgende Nachbesetzung gestützt werden. Die Arztstelle kann beim Ausscheiden dieses Arztes auch dann (erneut) nachbesetzt werden, wenn Zweifel daran bestehen, ob die erste Nachbesetzung hätte genehmigt werden dürfen, weil bei dem Leistungserbringer keine (volle oder anteilige) Arztstelle entstanden ist. ${ }^{26}$

Gesetzlich nicht geregelt ist, ob die Anstellungsgenehmigungen gemäß §95 Abs. 9 SGB V, die zuvor dem verzichtenden Vertragsarzt erteilt wurden, mit dem Verzicht zu Gunsten der Anstellung gemäß § 103 Abs. 4a S.1, 4b S. 1 SGB V auf den anstellenden Leistungserbringer übergehen bzw. neu erteilt werden können. Die Spruchpraxis der Zulassungsausschüsse ist unterschiedlich. Vorzugswürdig erscheint, dass durch die aufgrund des Zulassungsverzichts

24 BSG, Urteil vom 04.05.2016, Az.: B 6 KA 21/ 15 R, Rn. 29.

25 Müssig, Verzicht zum Zwecke der Anstellung, BSG schränkt Möglichkeiten für Ärzte und MVZ ein, KrV 2017, 16, 17.

26 BSG, Urteil vom 04.05.2016, Az.: B 6 KA 21/ 15 R, Rn. 33. erteilte Anstellungsgenehmigung der ursprüngliche Vertragsarztstatus vollumfänglich, d. h. mit den ihm zugeordneten Arztstellen, dem anstellenden Leistungserbringer zugeordnet wird. Der anstellende Leistungserbringer muss lediglich aus formellen Gründen für jeden angestellten Arzt des bisherigen Vertragsarztes eine eigene Anstellungsgenehmigung beantragen. ${ }^{27}$

Bei einem Verstoß gegen die Drei-JahresFrist ist fraglich, ob und inwieweit dies auch Auswirkungen auf die übergangenen sonstigen Arztstellen hat. Insbesondere der argumentative Ansatz des BSG spricht jedoch dafür, dass ein Verstoß gegen die Drei-jahres-Frist keine Auswirkungen auf diese Arztstellen hätte. ${ }^{28}$

\section{Ausnahmen von der Drei-Jahres-Frist}

Insbesondere im Rahmen der Ausnahmen von der Dreijahresfrist und deren Umfang und Tragweite ergibt sich erhebliche Rechtsunsicherheit. Daher sollen nachfolgend exemplarisch auf die möglichen Ausnahmen von der Dreijahresfrist eingegangen und zu noch ungeklärten Punkten Stellung genommen werden.

Nach dem BSG hängt das Nachbesetzungsrecht des MVZ davon ab, ob nach den Umständen davon ausgegangen werden kann, dass der ursprünglich zugelassene Arzt zunächst tatsächlich zumindest drei Jahre im MVZ tätig werden wollte, diese Absicht aber aufgrund von Umständen, die ihm zum Zeitpunkt des Verzichts auf die Zulassung noch nicht bekannt waren, nicht mehr realisieren konnte.

Das kann etwa der Fall sein, wenn er erkrankt oder aus zwingenden Gründen seine Berufsoder Lebensplanung ändern musste. ${ }^{29}$

Zum Teil wird daher angenommen, dass ein früheres Ausscheiden des Arztes dann unschädlich sei, wenn nach genehmigter

27 Kremer/Wittmann, Vertragsärztliche Zulassungsverfahren, 3. Auflage 2018, Rn. 901.

28 Pflugmacher, KrV 2017, 107, 113.

29 BSG, Urteil vom 04.05.2016, Az.: B 6 KA 21/ 15 R, Rn. 29.
Beschäftigung Umstände eintreten, die eine weitergehende Zusammenarbeit für die Vertragsparteien des Arbeitsvertrages nicht mehr zumutbar machen. Exemplarisch kann in diesem Zusammenhang auf die allgemeinen Gründe für eine außerordentliche Kündigung eines Arbeitsvertrages Bezug genommen werden. ${ }^{30}$ Gründe, die zu einer fristlosen Kündigung berechtigen, werden noch am ehesten unter die Formulierung des BSG der „zwingenden Gründe eine Änderung der Berufsplanung“ zu subsumieren sein. ${ }^{31}$

Erforderlich und ausreichend ist also, dass der Verzichtende zunächst die Absicht hatte, bei dem Vertragsarzt oder dem MVZ mindestens drei Jahre tätig zu sein und er diese Absicht im Verlauf seiner Angestelltentätigkeit auf Grund von besonderen Umständen nicht realisieren kann. Diese Umstände dürfen ihm zum Zeitpunkt des Verzichts nicht bekannt gewesen sein.

Maßstab der subjektiven Vorstellungen des verzichtenden Arztes ist demnach allein die positive Kenntnis von Umständen. Nach den Ausführungen des BSG ist davon auszugehen, dass insbesondere die fahrlässige oder grob fahrlässige Unkenntnis von Umständen im Hinblick auf das Nachbesetzungsrecht des anstellenden Leistungserbringers nicht schädlich ist.

Nach Auffassung des BSG gibt es jedoch auch bestimmte Indizien, die gegen eine Absicht zur mindestens dreijährigen Tätigkeit bei dem anstellenden Leistungserbringer sprechen. Wenn der Arzt im Zuge des Verzichts auf die Zulassung und der Beantragung der Anstellungsgenehmigung durch den Vertragsarzt oder das MVZ schon konkrete Pläne für die alsbaldige Beendigung seiner Tätigkeit entwickelt hat, spricht dies gegen eine Absicht der dreijährigen Tätigkeit. Hat der Leistungserbringer zum Zeitpunkt des Verzichts zu Gunsten der Anstellung schon Verhandlungen mit einem an der Nachbesetzung der betroffenen Arztstelle interessierten anderen Arzt geführt, die sich auf die unmittelbare Zukunft und nicht auf einen erst in drei Jahren beginnenden Zeitraum beziehen, so spricht

30 Schroeder/Printzen in: Ratzel/Luxemburger, Rn. 754.

31 Pflugmacher, KrV 2017, 107, 111. 
dies ebenso gegen einen Willen zur mindestens dreijährigen Tätigkeit. ${ }^{32}$

Die Anforderungen an den Nachweis der Umstände, die die Absicht zur Ausübung der Angestelltentätigkeit für eine Dauer von zumindest drei Jahren dokumentieren, hängen nach dem BSG von der tatsächlichen Dauer der Beschäftigung ab. Je kürzer die Angestelltentätigkeit des Arztes gewesen ist, desto höhere Anforderungen sind an den Nachweis der Umstände zu stellen. ${ }^{33}$ Einen sachlich durchgreifenden Grund für Abstufung des Umfangs der Nachweise bzw. der Beweiskraft derselben liefert das BSG nicht. Es ist auch vollkommen offen, inwieweit diese Abstufung tatsächlich vorzunehmen ist.

Nach Ansicht des BSG geht es zulasten des an der Nachbesetzung interessierten Vertragsarztes oder MVZ, wenn Änderungen der Verhältnisse, die eine Änderung der ursprünglich bestehenden Absichten nachvollziehbar erscheinen lassen, nicht festzustellen sind. ${ }^{34}$

Ob das BSG tatsächlich nur auf die Absicht des Verzichtenden und nicht auch sekundär auf die der MVZ-Trägergesellschaft oder des anstellenden Vertragsarztes abstellen wollte, ist fraglich. Denn soweit es diesen Rechtsgrundsatz formuliert hat, verwendet es auch im Wortlaut des Urteils den Plural „Absichten“. Wie oben ausgeführt sollen überdies Verhandlungen der MVZ-Trägergesellschaft mit einem anderen Arzt über die Nachbesetzung der Stelle ebenso für die Bewertung der Absicht des Verzichtenden relevant sein. ${ }^{35}$

In der Praxis nehmen Zulassungs- bzw. Berufungsausschüsse auf der Basis dieses Rechtsgrundsatzes an, dass hierdurch eine Art Beibringungsgrundsatz für den anstellenden Leistungserbringer in das Zulassungsverfahren implementiert worden sei.

Richtigerweise hat der Zulassungs- bzw. Berufungsausschuss gemäß $\S \S 45$ Abs. 3,

32 BSG, Urteil vom 04.05.2016, Az.: B 6 KA 21/ 15 R, Rn. 29.

33 BSG, Urteil vom 04.05.2016, Az.: B 6 KA 21/ 15 R, Rn. 29.

34 Ebd.

35 Pflugmacher, KrV 2017, 107, 111.
39 Abs. 1 Ärzte-ZV die ihm erforderlich erscheinenden Beweise zu erheben. Dies ist Ausdruck des Untersuchungsgrundsatzes des § 20 SGB X, der auch das Verfahren vor den Zulassungsgremien prägt. Der Zulassungsausschuss hat dementsprechend den Sachverhalt, d. h. alle für die zur Subsumtion unter die Tatbestandsmerkmale der einschlägigen Norm notwendigen Tatsachen, von Amts wegen zu ermitteln. Der Zulassungsausschuss muss alle Tatsachen ermitteln, die entscheidungserheblich („für den Einzelfall bedeutsamen “ Umstände, $\S 20$ Abs. 2 SGB X) sind. ${ }^{36}$ Hierzu gehören gemäß § 20 Abs. 2 SGB X auch die für die Beteiligten günstigen Umstände.

Aus dem Rechtsgrundsatz, dass nicht feststellbare Umstände zulasten des anstellenden Leistungserbringers gehen, ergeht daher allenfalls eine Feststellungslast ${ }^{37}$ (auch materielle Beweislast genannt) und entlastet die Zulassungsgremien nicht von der Erhebung sämtlicher erforderlichen Umstände.

Welche Fallkonstellationen unter die vom BSG darlegten Ausnahmen von der DreiJahres-Frist fallen, ist noch nicht abschließend geklärt. Vielmehr finden sich hierzu kaum Entscheidungen in der Sozialgerichtsbarkeit. In einem Urteil vom 30.09.2020 (Az.: S 87 KA 155/18) hatte das Sozialgericht (SG) Berlin jedoch eine Ausnahme von der Drei-Jahres-Frist anerkannt.

Dem Urteil lag folgender sehr vereinfachter Sachverhalt zugrunde: Eine Ärztin war 5 Jahre in Teilzeit in einer Arztpraxis tätig. Dies Arztpraxis wurde von einem MVZ erworben. Zur Sicherung ihrer Weiterbeschäftigung im MVZ wurde ihre Anstellungsgenehmigung mit halbem Versorgungsauftrag zunächst zu ihren Gunsten in eine Zulassung mit halbem Versorgungsauftrag umgewandelt. Nach einem halben Jahr Vertragsarzttätigkeit verzichtete sie (ohne finanzielle Gegenleistung) zugunsten einer Anstellung im MVZ. Bereits nach neun Monaten bekam die Ärztin ein Stellenangebot in einem konkurrierenden MVZ, welches eine Vollzeitanstellung in ihrem Fachbereich ermöglichte, was sie zu ihrer Kündigung bewog. Die Nachbesetzung

36 Ladurner, Ärzte-ZV, Zahnärzte-ZV, § 39 ÄrzteZV Rn. 2.

37 Ebenso: Pflugmacher, KrV 2017, 107, 112. wurde dem ursprünglichen Arbeitgeber der Ärztin wegen Nichterfüllung der DreiJahres-Frist sodann versagt. ${ }^{38}$

In seinem Urteil vom 30.09.2020 entschied das SG Berlin, dass die Nachbesetzung der Arztstelle der Ärztin dem MVZ nicht verwehrt werden dürfe. Maßgeblicher Zeitpunkt sei die Erklärung des Verzichts. Ausreichend sei die Absicht der Ärztin zu diesem Zeitpunkt gewesen, drei Jahre in dem MVZ tätig zu werden. Ein späteres Abrücken aufgrund von Umständen, welche zum Zeitpunkt des Verzichts noch nicht bekannt waren, sei unschädlich. Die beschriebene Aufstiegschance sei ein nachvollziehbarer und gewichtiger Grund für die Kündigung und zum Zeitpunkt des Verzichts für die Ärztin noch nicht absehbar gewesen. Außerdem sprächen weitere Umstände für diese Annahme, z. B. die Tatsache, dass die Ärztin bereits seit fünf Jahren in der Praxis tätig war. Ebenso spräche auch die vorherige Vorgehensweise der Umwandlung ihrer Anstellung in eine Zulassung und der anschließende Verzicht ohne jegliche finanzielle Gegenleistung hierfür. ${ }^{39}$

\section{Kritik an der Drei-Jahres- Frist}

Das Urteil des BSG vom 04.05.2016 (Az.: B 6 KA 21/15 R) ist in der Literatur erheblich kritisiert worden. ${ }^{40} \mathrm{Im}$ Wesentlichen wurde an diesem Urteil kritisiert, dass es die Grenzen der richterlichen Rechtsfortbildung erheblich ausgedehnt, wenn nicht sogar überschritten habe und insbesondere die konkrete Dauer und Festsetzung der Dreijahresfrist nicht nachvollziehbar sei.

Ein weiterer kritikwürdiger Ansatz ist, dass die vom 6. Senat zu $§ 103$ Abs. 4a SGB V entwickelte Rechtsprechung zur Mindestbeschäftigungsdauer - gestützt vor allem

38 Kuhlmann/Stratenhoff, MVZ Update Vertragsarztrecht, fw 2021, 636.

39 Ebd.

40 Plagemann/Bergmann, DStR 2017, 1392 „Tendenz ... bedenklich“; Willaschek, MedR 2016, 1011 ff. (, Rechtsfortbildung contra legem“); Greve, ZMGR 2016, $379 \mathrm{ff}$; Kuhlmann, ZMGR 2018, 3, 4: „weit über das gesetzgeberische Ziel hinausgeschossen “. Die geforderte Dauer von drei Jahren erscheine "willkürlich und als freie Rechtsschöpfung ohne gesetzliche Grundlage“. 
auf Überlegungen zur Unterbindung des Handels mit Zulassungen - nicht ausreichend konsistent erscheint. Denn der 6. Senat habe die Privilegierung der „verbleibenden “ Vertragsärzte in einer BAG gem. §103 Abs. 6 S. 2 SGB V - die im Ergebnis ebenfalls zu einem Zulassungshandel führen könnte - gerade nicht an eine feste Mindestdauer der gemeinschaftlichen Ausübung der vertragsärztlichen Tätigkeit in der BAG gebunden. ${ }^{41}$ Der Senat begründete dies im Hinblick auf die BAG damit, dass eine solche Mindestzeit im Gesetz „nicht vorgesehen“ sei. Es sei allein Sache des Gesetzgebers, eine Mindestzeit für die Privilegierung solcher Kooperationen im Rahmen der Auswahlentscheidung zu schaffen. ${ }^{42}$

Dass bei der Privilegierung der BAG in Nachbesetzungsverfahren eine Regelung wie $\S 103$ Abs. 3a Satz 5 SGB V indes nur vom Gesetzgeber geschaffen werden kann, bei § 103 Abs. 4a Satz 1 SGB V dagegen richterrechtlich ein Rückgriff auf den Rechtsgedanken des $§ 103$ Abs. 3a Satz 5 SGB V geboten sein soll, vermag nicht zu überzeugen. Dem Wortlaut des $§ 103$ Abs. 4a Satz 1 SGB $\vee$ lässt sich ebenso wenig wie dem Wortlaut § 103 Abs. 6 Satz 2 SGB V ein Hinweis auf den zeitlichen Umfang der Tätigkeit in der neuen Kooperation entnehmen. In beiden Fällen hat der Gesetzgeber gleichermaßen auf die Festlegung einer Mindesttätigkeitsdauer verzichtet. ${ }^{43}$

Teilweise wird hierzu angemerkt, dass die insoweit geltend gemachte Inkonsistenz der Rechtsprechung nicht gegeben sei. Denn der 6. Senat habe entschieden, dass auch bei einer BAG eine sehr geringe Dauer des Betriebs derselben von erheblicher Bedeutung sein könne. Daneben seien die Folgen einer Beeinflussung des Nachbesetzungsverfahrens in einer BAG andere als bei der Umgehung des Auswahlverfahrens durch Übernahme des Vertragsarztsitzes im Wege des Verzichts zu Gunsten der Anstellung. Hierdurch könne eine Zementierung von Überversorgung eintreten. Daneben sei zu befürchten, dass in Einzelfällen

41 Ladurner/Walter/Jochimsen, Gutachten MVZ, S. 69.

42 BSG, Urt. v. 22.10.2014, Az.: B 6 KA 44/13 R, Rn. 46.

43 Ladurner/Walter/Jochimsen, Gutachten MVZ, S. 69. qualifizierte Niederlassungswillige kleinerer Arztgruppen keine Niederlassungsmöglichkeit hätten, auch wenn sie sich in jedem Auswahlverfahren durchsetzen würden. ${ }^{44}$

Zwar ist der Auffassung grundsätzlich zuzustimmen, dass die Beeinflussung des Nachbesetzungsverfahrens durch die Gründung einer BAG und die Umgehung des Nachbesetzungsverfahrens durch den Verzicht zu Gunsten der Anstellung unterschiedliche Folgen haben. Dies mag jedoch nicht darüber hinweghelfen, warum der 6 . Senat in einer Entscheidung ausdrücklich annimmt, dass es Aufgabe des Gesetzgebers sei, eine Mindestzeit für Privilegierung vorzusehen, um eine solche Mindestzeit dann in einer anderen Entscheidung - nämlich beim Verzicht zu Gunsten der Anstellung - im Rahmen der richterlichen Rechtsfortbildung vorzusehen. Der 6. Senat überschreitet mithin sich selbst gesetzte Grenzen anschließend wieder.

Es ist zwar anzuerkennen, dass insoweit eine zumindest missbrauchsanfällige Rechtslage besteht. ${ }^{45}$ Die rechtliche Lösung sollte jedoch nicht im Rahmen des Richterrechts gesucht werden. Vielmehr sollte der parlamentarische Gesetzgeber diese grundrechtswesentliche Entscheidung selbst treffen. In Betracht käme daher de lege ferenda die gesetzliche Umsetzung der Rechtsprechung des BSG mit dreijähriger Mindesttätigkeitsfrist bei gleichzeitigem Vorsehen mehrerer Ausnahmen von der Mindestfrist und der Reduktionsmöglichkeit im Alter. ${ }^{46}$ Ebenso könnte man lediglich eine einjährige Mindestfrist vorsehen und auf eine umfangreiche Regelung von Ausnahmetatbeständen verzichten. ${ }^{47}$ Vorteil der zweiten Variante ist die einfachere Handhabbarkeit und dass diese regelmäßig weniger streitanfällig sein wird. Ebenso ist fraglich, ob Mindesttätigkeitsvorgaben für die Versorgungsqualität und Motivation der angestellten Ärzte förderlich sind. ${ }^{48}$
44 Wenner, SGb 2021, 593, 602.
45 Wenner, SGb 2021, 593, 602.
46 Ladurner/Walter/Jochimsen, Gutachten MVZ, S. 69.
47 Ladurner/Walter/Jochimsen, Gutachten MVZ S. 72.
48 Ladurner/Walter/Jochimsen, Gutachten MVZ, S. 73 .

\section{Zusammenfassung}

Verzichtet ein Vertragsarzt gemäß $§ 103$ Abs. 4a S. 1, 4b S. 1 SGB V, um bei einem anderen Vertragsarzt oder MVZ tätig zu werden, hängt das Nachbesetzungsrecht des anstellenden Leistungserbringers nach dem Ausscheiden des Verzichtenden davon $a b$, ob der Verzichtende mindestens drei Jahre bei dem anstellenden Leistungserbringer tätig war.

Ist dies der Fall, spricht dies dafür, dass der Vertragsarzt auf seine Zulassung verzichtet hat, um gerade bei diesem Leistungserbringer als angestellter Arzt tätig zu werden. Scheidet der Arzt jedoch vor Ablauf der dreijährigen Tätigkeit aus dem Arbeitsverhältnis aus, ist nach den Grundsätzen des BSG die Absicht des Verzichtenden zum Zeitpunkt des Verzichts ausschlaggebend. Der Verzichtende muss die Absicht gehabt haben, mindestens drei Jahre bei dem Leistungserbringer tätig zu sein.

Im Rahmen der Nachbesetzung der Arztstelle hat der Zulassungsausschuss anhand der von der Rechtsprechung aufgezeigten Indizien zu prüfen, ob der Vertragsarzt diese Absicht zum Zeitpunkt des Verzichts hatte. Daneben darf er diese Absicht aufgrund von Umständen, die ihn zum Zeitpunkt des Verzichts nicht positiv bekannt waren, nicht realisiert haben können. Die Feststellungslast für die aufgezeigten Punkte trägt der nachbesetzungswillige Vertragsarzt oder das MVZ.

Altersbedingt können jedoch die verzichtenden Ärzte den zeitlichen Umfang ihrer Tätigkeit schrittweise reduzieren. Hierzu müssen sie jedoch ein Jahr in dem Umfang, mit welchem sie zuvor als zugelassener Vertragsarzt an der Versorgung teilgenommen hatten, als angestellter Arzt bei dem anstellenden Leistungserbringer tätig gewesen sein.

Objektiv ergibt sich eine zumindest missbrauchsanfällige Rechtslage und eine Ungleichbehandlung zum Nachbesetzungsverfahren. Die Lösung für diesen Umstand sollte jedoch nicht allein und nicht in diesem Umfang durch richterliche Rechtsfortbildung erfolgen. Vielmehr sollte der Gesetzgeber diese grundrechtlich relevanten Entscheidungen selbst treffen. 
Trotz der durch das BSG gestellten Anforderungen an die Nachbesetzbarkeit eine Arztstelle, die durch einen Verzicht zu Gunsten Anstellung bei einem Vertragsarzt oder MVZ nach $\S 103$ Abs. 4a S. 1, 4b S. 1 SGB $V$ entstanden ist, stellt der Verzicht zu Gunsten Anstellung nach wie vor die überwiegende Option in der Praxis dar, eine vertragsärztliche Zulassung und die Arztpraxis als solche auf eine andere Rechtsperson zu übertragen. Hierbei ist jedoch darauf zu achten, dass die Einhaltung der Vorgaben des BSG vertraglich sichergestellt werden.
Prof. Dr. Peter Wigge

Rechtsanwalt

Fachanwalt für Medizinrecht

Hendrik Hörnlein

Rechtsanwalt

Rechtsanwälte Wigge

Scharnhorststraße 40

48151 Münster

Telefon: (0251) 53 595-0

Telefax: (0251) 53 595-99

E-Mail: kanzlei@ra-wigge.de www.ra-wigge.de 